\title{
Método Francês versus método Brasileiro para avaliar a DEF em concreto.
}

\author{
R.H.R.Q. Melo ${ }^{1 *}$, D. Schovanz ${ }^{2}$, F. Tiecher ${ }^{3 *}$, N. P. Hasparyk ${ }^{4 *}$, S. C. Kuperman ${ }^{5}$ \\ *Melo: rodrigohquevedo@gmail.com \\ *Hasparyk: nicole@furnas.com.br \\ *Tiecher: Francieli.bonsembiante@imed.edu.br
}

\author{
${ }^{1}$ Master student in Civil Engineering of Faculdade Meridional - IMED, Passo Fundo, Brazil \\ ${ }^{2}$ Master, PhD student in Civil Engineering of Universidade Federal do Rio Grande do Sul - UFRGS, Porto Alegre, Brazil \\ ${ }^{3} \mathrm{PhD}$, Professor in Civil Engineering of Faculdade Meridional - IMED, Passo Fundo, Brazil \\ ${ }^{4} \mathrm{PhD}$, Researcher of Departamento de Segurança de Barragens e Tecnologia, ELETROBRAS Furnas, Goiânia, Brazil \\ ${ }^{5} \mathrm{PhD}$ in Civil Engineering - DESEK, São Paulo, Brazil
}

\begin{abstract}
RESUMO
Este trabalho apresenta o comportamento de concretos submetidos a dois diferentes métodos de ensaio a fim de avaliar o potencial para a DEF (Métodos Francês e Brasileiro). Concretos foram moldados e submetidos a cura térmica até $85^{\circ} \mathrm{C}$, sendo a principal diferença entre os métodos o ambiente de exposição ao longo do tempo. Ensaios mecânicos e de expansão, além de análises microestruturais foram realizados a fim de verificar o nível de dano a partir das expansões de DEF atingidas. Os resultados obtidos indicaram comportamentos distintos entre os métodos testados. Para a idade de seis meses de ensaio, o método brasileiro se mostrou mais acurado para avaliar a DEF, considerando o cimento testado responsável por essa patologia em campo.
\end{abstract}

Palavras-chave: DEF; Expansão; Concreto; Método Francês; Método Brasileiro. 


\begin{abstract}
This paper presents results from an experimental program into the potential concrete behavior related to DEF by the use of two test methods (French and Brazilian). Concrete specimens were cast and undergone thermal curing at $85^{\circ} \mathrm{C}$. The main difference between the methods was the exposure condition over time of specimens after cured. Mechanical and expansion tests, besides microstructure analyses were performed in order to evaluate the level of damage caused by DEF over time. The results indicate different behaviors depending on the method used. At six months, the Brazilian test performed better to identify the concrete potential to DEF, in the presence of the cement with no mineral admixture.
\end{abstract}

Keyword: DEF; Expansion; Concrete; French method; Brazilian method.

\title{
RESUMEN
}

Este trabajo presenta el comportamiento de hormigones sometidos a dos métodos de ensayo para evaluar el potencial de DEF (método francés y brasileño). El hormigón fue moldeado y sometido a curado térmico hasta $85^{\circ} \mathrm{C}$, siendo la principal diferencia entre los métodos el ambiente de exposición a lo largo del tiempo. Se realizaron ensayos mecánicos y de expansión, así como análisis microestructurales con el fin de verificar el nivel de daño por las expansiones de DEF alcanzadas. Los resultados obtenidos indicaron diferentes comportamientos entre los métodos probados. Para la edad de seis meses de prueba, el método brasileño demostró ser más preciso para evaluar DEF, considerando el cemento probado responsable de esta patología en el campo.

Palabras clave: DEF; Expansión; Hormigón; Método Francés; Método Brasileño. 


\section{INTRODUÇÃO}

A formação da etringita tardia (DEF) é um fenômeno que ocorre em elementos de concreto já endurecidos, sendo um dos tipos de ataques por sulfato interno (ISA), responsável pela degradação de elementos de concreto. Para que a DEF ocorra são necessários alguns fatores, sendo os principais relacionados à composição química do cimento, que é a fonte interna de íons sulfato, à temperatura de cura, superior a $60^{\circ} \mathrm{C}$, e à presença de água. Fatores como esses podem deflagar esta reação nociva, levando a expansões e consequentemente a fissurações no elemento de concreto que, aos poucos, vão realimentando a formação destas reações, e reduzindo a capacidade mecânica e a durabilidade da estrutura (Hasparyk e Kuperman, 2019; Schovanz et al., 2021; Jebli et al., 2021).

Os mecanismos da DEF são complexos quando comparados à outras manifestações patológicas que ocorrem no concreto; além disso, a avaliação do potencial de ocorrência da DEF em elementos de concreto não possui normas ou testes estabelecidos por órgãos regulamentadores ou entidades de classe (Schovanz, 2019). Há, no entanto, algumas metodologias laboratoriais, que foram publicadas buscando encontrar as melhores maneiras de avaliar as condicionantes e mensurar o potencial da formação de etringita tardia, destacando-se um método francês e um brasileiro.

Entre os métodos encontrados na literatura cita-se o "Duggan test", que foi desenvolvido para uma análise acelerada do potencial de reatividade álcali-agregado, mas, posteriormente, foi empregado para a avaliação do potencial para DEF, nos estudos de Day (1992), Grabowski et al. (1992) e Torres e Andrade (2016). Trata-se de um procedimento que demanda pouco tempo de ensaio, cuja concepção é de Duggan e Scott (1987), porém, Gillott et al. (1989) atribuíram à DEF a principal causa de expansão de concretos expostos a este teste. Vale destacar a preocupação com o tamanho bastante reduzido da amostra ensaiada, bem como o curto tempo do ensaio (20 dias).

O Laboratório Central de Pontes e Estradas, da França (Laboratoire Central des Ponts et Chaussées), publicou um método para avaliar a capacidade de um concreto suportar uma reação interna de sulfato devido à DEF. $\mathrm{O}$ método de teste $\mathrm{n}^{\circ} 66$ do $\mathrm{LCPC}$, denominado neste trabalho como método francês, é aplicável a concretos que venham a sofrer elevação de temperatura a patamares superiores a $65^{\circ} \mathrm{C}$, além de estarem expostos a ambiente úmido (LCPC, 2007), tendo sido o método utilizado nas pesquisas de Pavoine et al. (2006), Lavaud et al. (2013), Godart et al. (2013) e Godart (2017). O método prevê que, após um ano de ensaio, o limite adotado para a expansão média máxima seja de $0,04 \%$, ou para as expansões individuais, de $0,06 \%$, para considerar o concreto com potencial para o desencadeamento da DEF (LCPC, 2007).

Mais recentemente, e por meio de um projeto de P\&D ANEEL, Furnas realizou um amplo programa de estudos sobre reações expansivas, no qual foi possível avaliar DEF através de variados métodos e compósitos. Em Hasparyk et al. (2020) está contido um procedimento elaborado por este projeto e que propõe uma nova metodologia, onde a cura definida foi considerada uma das etapas fundamentais a fim de propiciar a elevação da temperatura nas primeiras horas da hidratação do cimento, além do ambiente de exposição, fornecendo condições propícias para a ocorrência da DEF, caso o concreto tenha esse potencial. Essa metodologia foi denominada neste trabalho como método brasileiro, e deu origem à instrução técnica intitulada "Método de ensaio para a avaliação do potencial de ocorrência da etringita tardia (DEF) em concreto" (Hasparyk et al., 2020). O método brasileiro já foi empregado nas pesquisas de Schovanz (2019), Langoski et al. (2020) e Oliveira et al. (2020).

Kchakech et al. (2016), Giannini et al. (2018), Sanchez et al. (2018) e Bronholo (2020) utilizaram procedimentos semelhantes ao método brasileiro, entretanto, no momento do tratamento térmico as amostras não se encontravam imersas em água.

Existem outras semelhanças entre as metodologias laboratoriais, como por exemplo o tratamento térmico inicial, com temperaturas sempre superiores a $60^{\circ} \mathrm{C}$ para promover o desenvolvimento da $\mathrm{DEF}$ o longo período de duração do ensaio. Outro ponto comum a todos os métodos é a manutenção das amostras em ambientes de cura úmida durante e após os ciclos térmicos, pois a presença de água, ou elevada umidade, é outro fator fundamental para a ocorrência da DEF, uma vez que as reações químicas e as trocas iônicas ocorrem no interior dos poros ou fissuras saturadas (Leklou, 2008; Godart et al., 2013). 
a DEF, apenas publicações a nível internacional, e poucas publicações nacionais, nas quais cada pesquisador adota um procedimento, e o método de ensaio do LCPC (método francês), esta pesquisa foi proposta, e apresenta os resultados obtidos a partir do emprego do método brasileiro e do método francês na avaliação do potencial para a DEF.

\section{PROGRAMA EXPERIMENTAL}

\subsection{Materiais e dosagem dos concretos}

Os materiais empregados no estudo experimental foram os seguintes:

- Cimento de alta resistência inicial (Tipo CP V- ARI, Brasileiro).

- Agregados miúdo e graúdo de origem granítica

- Aditivo polifuncional à base de lignosulfonato e policarboxilato

As principais características químicas do cimento empregado, em \%, são: $\mathrm{CaO}: 61,75 ; \mathrm{SiO}_{2}: 18,89$; $\mathrm{Fe}_{2} \mathrm{O}_{3}: 3,00 ; \mathrm{Al}_{2} \mathrm{O}_{3}: 4,43 ; \mathrm{MgO}: 2,37 ; \mathrm{Na}_{2} \mathrm{O}_{\text {eq. }}: 0,80$ e $\mathrm{SO}_{3}: 2,74$. A perda ao fogo é 3,59\% e o resíduo insolúvel igual a 0,7\%. Os agregados miúdo e graúdo possuem módulo de finura de 3,11 e 6,82, respectivamente.

A produção dos concretos seguiu as orientações contidas na NBR 5738 (ABNT, 2016). Foram confeccionados corpos de prova cilíndricos $(10 \mathrm{~cm} \times 20 \mathrm{~cm})$ e prismáticos $(7,5 \mathrm{~cm} \times 7,5 \mathrm{~cm} \times 28,5$ $\mathrm{cm})$, sendo o traço do concreto adotado de 1:1,6:1,9:0,46.

\subsection{Métodos de ensaio para indução da DEF}

O processo do tratamento térmico foi dividido em quatro etapas, segundo Schovanz (2019) e Hasparyk et al. (2020), a saber:

- Pré-cura: em situação semelhante à de câmara úmida por seis horas;

- Aquecimento: imersão em água com taxa de $10^{\circ} \mathrm{C} / \mathrm{h}$ até $85^{\circ} \mathrm{C}$;

- Período isotérmico: manutenção em $85^{\circ} \mathrm{C}$ por 12 horas

- Resfriamento: taxa de $10^{\circ} \mathrm{C} / \mathrm{h}$ até a temperatura indicada para cada um dos métodos.

Após o final do processo de cura térmica descrito, os corpos de prova foram submetidos a dois diferentes ambientes de exposição, conforme Tabela 1, sendo mantidos por um período de seis meses, inferior ao indicado nos referidos métodos.

Tabela 1. Detalhes das condições de ensaio dos métodos Francês e Brasileiro.

\begin{tabular}{|c|c|c|}
\hline Condição & $\begin{array}{c}\text { Francês } \\
\text { (LCPC, 2007) }\end{array}$ & $\begin{array}{c}\text { Brasileiro } \\
\text { (Hasparyk et al., 2020) }\end{array}$ \\
\hline $\begin{array}{c}\text { Temperatura a ser } \\
\text { atingida no resfriamento }\end{array}$ & $20^{\circ} \mathrm{C}$ & $38^{\circ} \mathrm{C}$ \\
\hline \multirow{2}{*}{$\begin{array}{l}\text { Ambiente após } \\
\text { resfriamento }\end{array}$} & $\begin{array}{l}\text { Ciclo de secagem: } 7 \text { dias } \\
\text { Câmara climática a } 38^{\circ} \mathrm{C} \text { e UR }<30 \%\end{array}$ & \multirow{4}{*}{$\begin{array}{l}\text { Manutenção a } 38^{\circ} \mathrm{C} \text { com } \\
\text { imersão em água }\end{array}$} \\
\hline & $\begin{array}{l}\text { Ciclo de molhagem: } 7 \text { dias } \\
\text { Imersão em água a } 20 \pm 2{ }^{\circ} \mathrm{C}\end{array}$ & \\
\hline Observação & $\begin{array}{l}\text { Os concretos devem ser submetidos a dois ciclos } \\
\text { de secagem e molhagem seguidos (total: } 28 \text { dias) }\end{array}$ & \\
\hline Ambiente de exposição & $\begin{array}{l}\text { Armazenamento em tanque de água com } \\
\text { temperatura de } 20 \pm 2^{\circ} \mathrm{C}\end{array}$ & \\
\hline
\end{tabular}

Nota: A duração recomendada tanto no método Francês quanto no Brasileiro é de 12 meses.

\subsection{Ensaios e investigações realizados}

Ao longo do tempo, corpos de prova cilíndricos foram sendo retirados do ambiente de exposição de forma a determinar as propriedades mecânicas dos concretos ao longo de seis meses (resistência à compressão e à tracão), seguindo as normas da ABNT NBR 5739 (2018) e NBR 7222 (2011), além do 
(2021). Ainda, periodicamente e até a idade de seis meses, os comprimentos dos prismas de concreto também foram sendo monitorados, de forma a avaliar o potencial de expansão ocasionado pela DEF. Por fim, análises por microscopia eletrônica de varredura foram realizadas na idade de seis meses para uma avaliação comparativa da microestrutura dos concretos submetidos aos dois diferentes métodos de exposição.

\section{RESULTADOS}

De acordo com a variação dimensional dos prismas de concreto, foi possível verificar que os concretos com o cimento CP V-ARI, submetidos ao método brasileiro, expandiram de forma gradativa e expressiva, atingindo, em média, cerca de 1\% aos 6 meses. Por outro lado, o concreto ensaiado pelo método francês apresentou expansões bem inferiores, resultando em um percentual médio em torno de $0,05 \%$ na mesma idade (Figura 1 ).

É possível observar um grande afastamento no nível de expansões entre os dois métodos empregados. Na idade de 6 meses, o concreto ensaiado pelo método francês representa, em termos percentuais, apenas aproximadamente $5,2 \%$ das expansões obtidas com o método brasileiro. Considerando que o ciclo térmico adotado foi o mesmo, as diferenças decorrem do ambiente de exposição em cada um dos procedimentos. Enquanto no método brasileiro a temperatura é de $38^{\circ} \mathrm{C}$, no francês a usada é de $20^{\circ} \mathrm{C}$. Por outro lado, no método francês são realizados ciclos de secagem e molhagem, de forma a agravar ou mesmo acelerar o processo da DEF; entretanto, o que parece ter ocorrido foi a lixiviação dos íons sulfato, por meio dos ciclos, mascarando os resultados dos ensaios e reduzindo o nível das expansões atingidas. Uma continuidade dos estudos nesse sentido, em termos de análises químicas e outras técnicas complementares, devem ser realizadas para comprovar essa hipótese, ou definir as reais causas das diferenças tão expressivas obtidas.

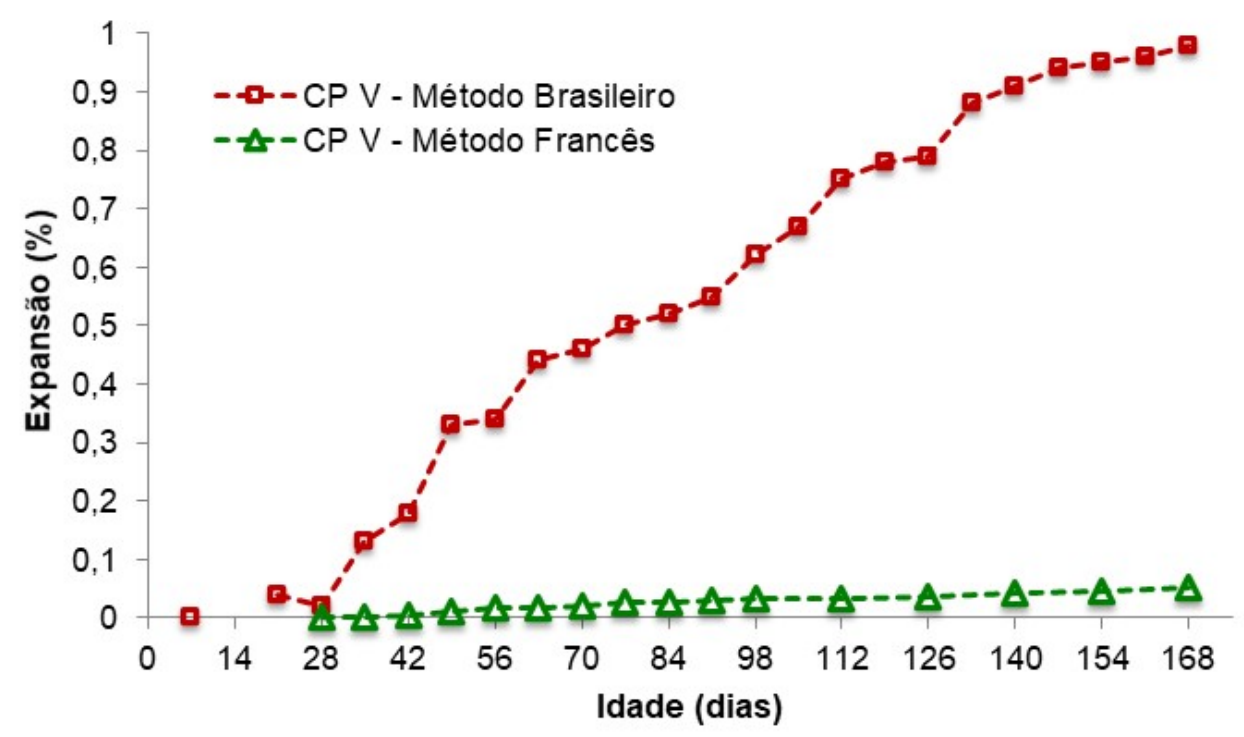

Figura 1. Expansões dos prismas de concreto ao longo do tempo.

Já se sabe que as neoformações de cristais de etringita passam a preencher os interstícios do concreto, propiciando um aumento volumétrico no elemento, o que ocasionará a expansão. Alguns pesquisadores (Martin et al. 2017 e Giannini et al. 2018) relataram encontrar expansões próximas a 1\% aos seis meses de estudos utilizando um método de cura térmica semelhante ao método brasileiro, o que corrobora aos resultados encontrados, além de indicar alta probabilidade de esta expansão ser devida à DEF.

Brunetaud (2005) propôs um esquema global da deterioração do concreto afetado por DEF, dividiu o fenômeno em período latente, onde há pouca atividade aparente e a expansão é lenta; período de aceleração da deterioração, onde ocorre a expansão substancial do elemento, com o preenchimento dos poros e da interface pasta-agregado por cristais de etringita comprimidos; e período de estabilização, onde as reações diminuem, desacelerando o inchamento do elemento. Essa curva sigmoide representando a expansão ocasionada pela DEF também foi adotada por Pichelin et al. (2020), que visualizou aue n neríndo de latência node ser mainr nu menor de acordo com as fiscuras oeradas na 
zona de transição durante o tratamento térmico, $\overline{\text { pois, com poucas fissuras nessa região o volume de }}$ DEF necessário para gerar pressão é reduzido, levando a uma diminuição do período de latência.

Os resultados de resistência à compressão e de resistência à tração indicaram uma expressiva e gradativa queda dessas propriedades a partir dos 28 dias para o concreto submetido ao método brasileiro (Figura 2 e 3). Comparando os dois métodos empregados, enquanto no método brasileiro a redução foi da ordem de 50\% para resistência à compressão e à tração, no método francês observaram-se ganhos para essas resistências de aproximadamente $40 \%$ e $20 \%$, respectivamente.

A resistência à tração é, em geral, uma propriedade mais sensível aos danos ocasionados por reações expansivas, como a DEF, quando comparada à resistência à compressão. Alguns autores explicam que isso se deve ao fato de os cristais de etringita estarem presentes nos interstícios da estrutura da matriz cimentícia, que resiste em parte à força compressiva, por meio da colmatação dos poros (Sanchez et al., 2018; Thiebaut et al., 2018; Schovanz, 2019).

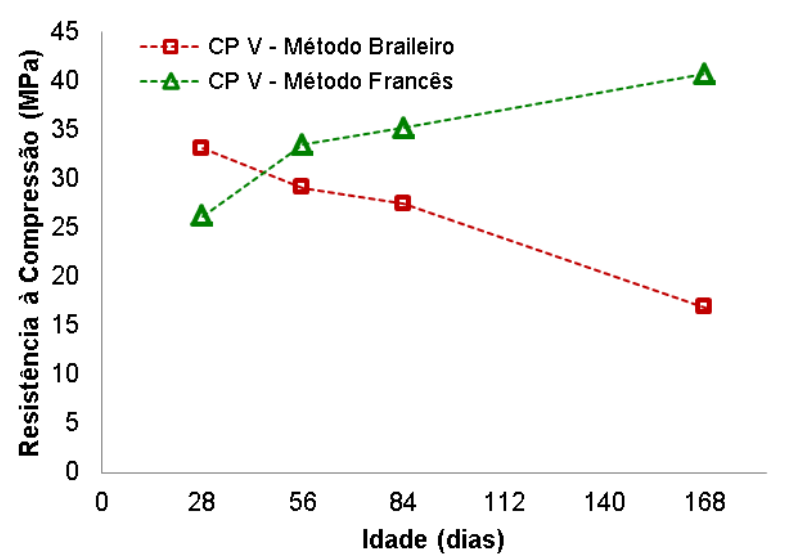

Figura 2. Resistência à compressão ao longo do tempo.

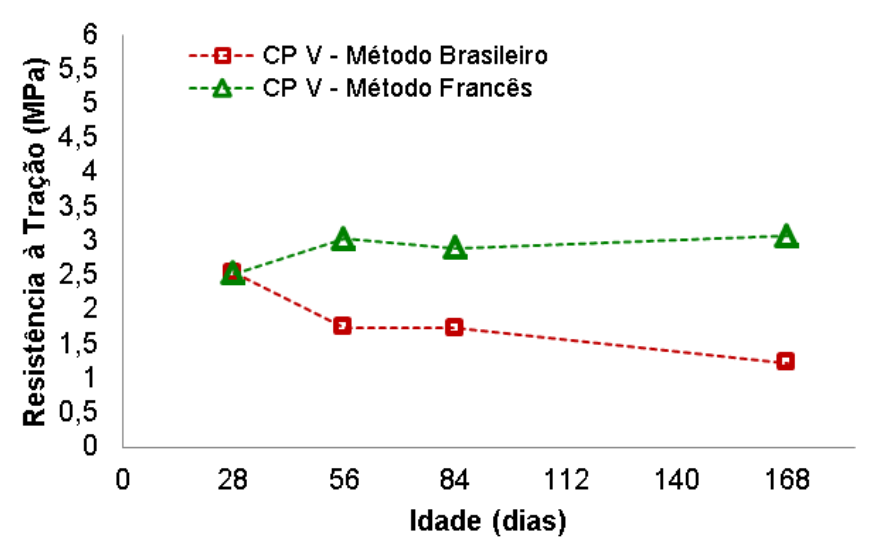

Figura 3. Resistência à tração ao longo do tempo.

As Figuras 4 e 5 retratam a relação entre os resultados médios da resistência à compressão e da resistência à tração perante a expansão, para os métodos brasileiro e francês, respectivamente. $O$ gráfico para o método brasileiro apresentou comportamento inversamente proporcional, ou seja, enquanto o percentual de expansão se elevou, as resistências apresentaram redução. Para o método francês o que observou-se uma menor sensibilidade à DEF, uma vez que as resistências aumentaram ao longo do tempo, assim como a expansão, embora em níveis bem inferiores àquelas obtidas no método brasileiro. Segundo Melo (2010), Leklou et al. (2016) e Jebli et al. (2021), a resistência à compressão não costuma ser uma propriedade muito sensível à DEF, principalmente quando se trata de baixos níveis de expansão, apresentando interferências negativas significantes apenas após elevada expansão e consequente fissuração das amostras, fato que corrobora com os resultados encontrados nesta pesquisa.

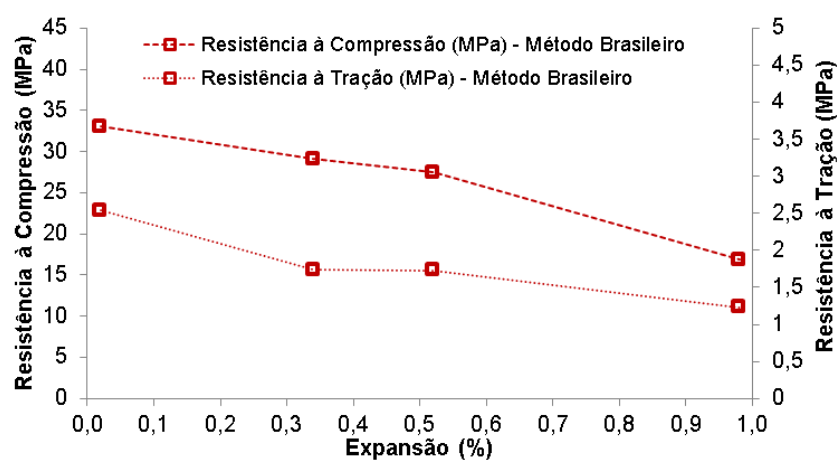

Figura 4. Comportamento mecânico x expansão método brasileiro.

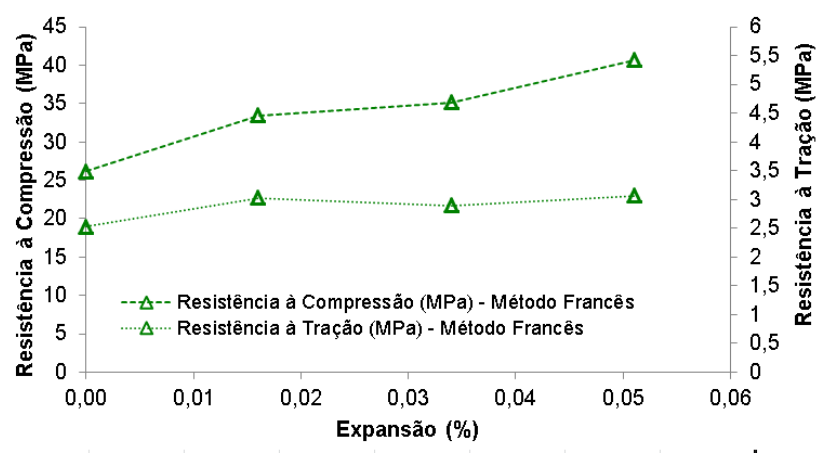

Figura 5. Comportamento mecânico x expansão - método Francês.

Os ensaios realizados aos 180 dias pelo método SDT mostraram também comportamentos diferenciados entre os métodos, conforme pode ser observado na Figura 6 e 7. 


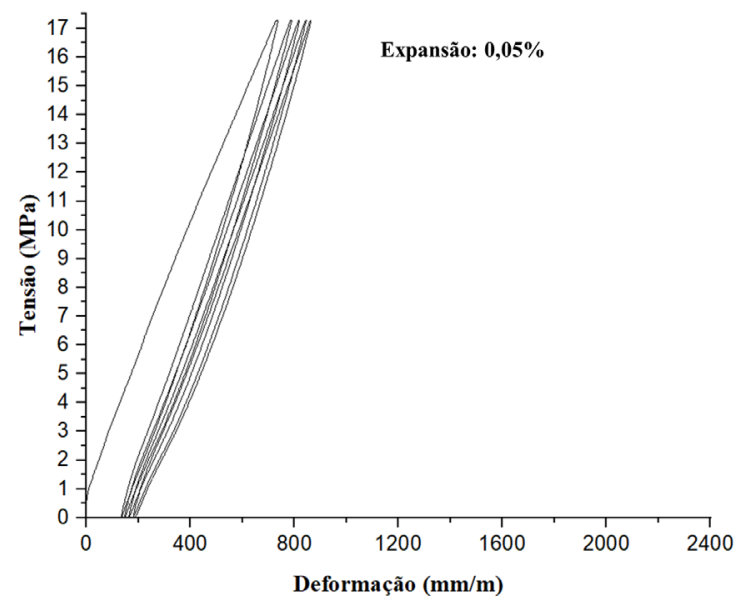

a)

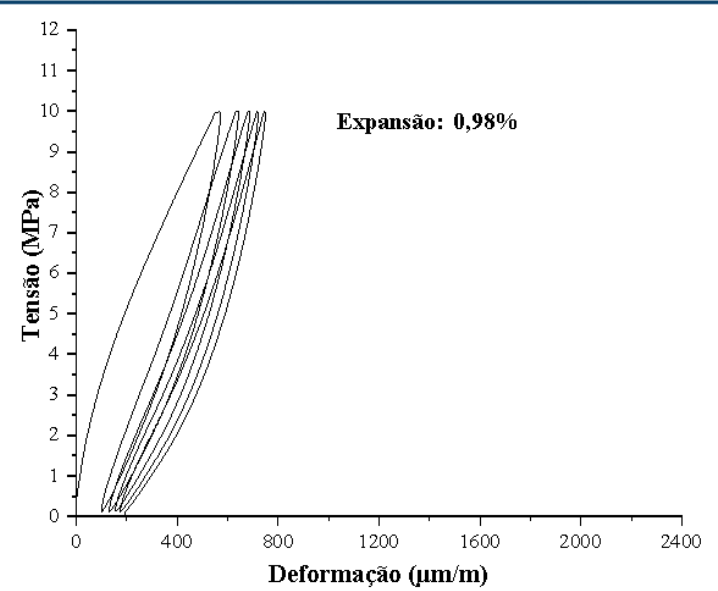

b)

Figura 6. Diagrama tensão-deformação obtido pelo SDT: a) Método francês; b) Método brasileiro.

Através do ensaio do SDT são obtidos os valores de Índice de Dano de Rigidez (SDI) e Índice de Deformação Plástica (PDI), que representam, respectivamente, a razão de energia dissipada/deformação plástica e à energia total/deformação implementada no sistema. Estes parâmetros estão apresentados na Figura 7.

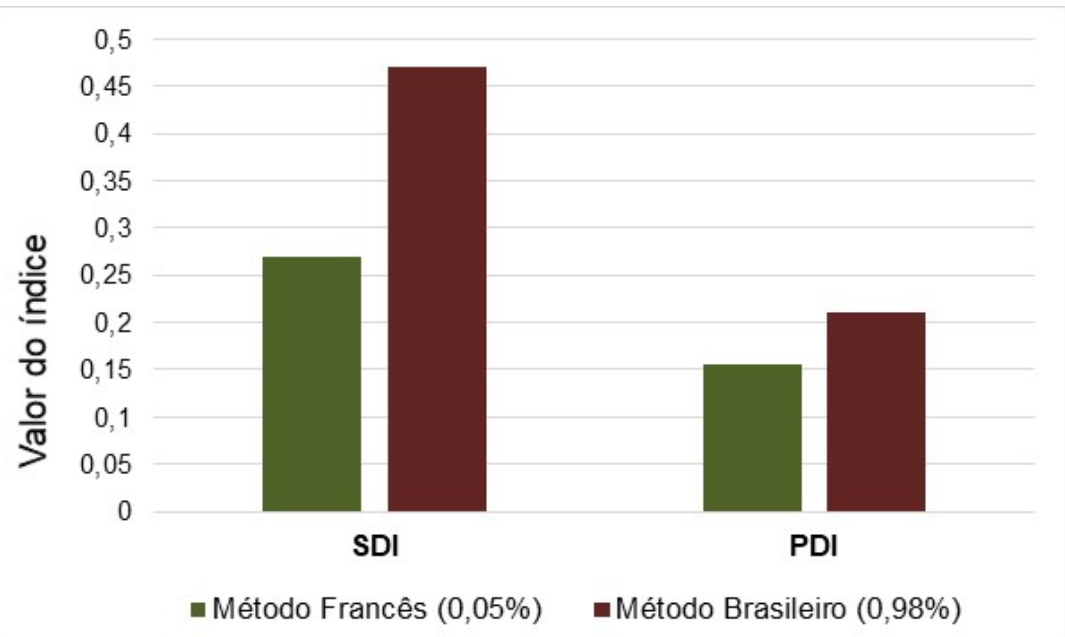

Figura 7. SDI e PDI obtidos aos 180 dias pelo SDT para os concretos submetidos ao método brasileiro e francês.

As amostras do método francês apresentaram valores de SDI próximos à 0,25 para expansão de $0,05 \%$, enquanto as amostras do método brasileiro com expansão de $0,98 \%$ resultaram em maiores valores de SDI, superiores à 0,45 que representam a perda da integridade física deste elemento. Estes resultados estão de acordo com os encontrados por Giannini et al. (2018), onde os valores de SDI variaram de 0,14 a 0,46 , e os valores de PDI de 0,13 a 0,42 , para níveis de expansão entre $0 \%$ e $1,01 \%$, assim como os resultados encontrados por Martin et al. (2017) e Sanchez et al (2018), para níveis de expansão maiores que $0,50 \%$. O parâmetro PDI indica a quantidade de fissuras internas em um material que foi danificado por meio da medição de sua energia dissipada durante o ensaio de SDT (Sanchez et al., 2017; 2020), conforme os níveis de expansão aumentam, mais sinais de danos podem ser vistos nos valores de SDI apresentados no método brasileiro, que foram bastante superiores ao método francês (Figura 7).

Aos 180 dias amostras dos concretos ensaiados nos dois métodos foram selecionadas para investigação por MEV/EDS. As imagens a seguir mostram as diferenças microestruturais dos concretos submetidos aos dois métodos. Conforme verificado por meio das maiores expansões atingidas, a concentração dos cristais de etringita neoformada nos poros e na pasta é muito mais marcante no concreto testado pelo método brasileiro (Figura 8). Esse maior volume de etringita é efetivamente o responsável pelas altas taxas de expansão. Ainda, a formação de cristais massivos na zona de transição pasta/agregado e a 
pelo MEV, indica as altas tensões geradas e as fissurações observadas a nível microestrutural, sendo essa microfissuração a responsável pelas quedas das resistências à compressão e à tração. Em relação à rigidez do material, apresentada anteriormente, essa se mostrou muito mais afetada pelas fissurações nesses concretos, conforme índices SDI e PDI.

Nos concretos submetidos ao método francês, a microestrutura evidenciou alguns poros vazios, sem neoformações facilmente visíveis nas zonas de transição (Figura 9). Contudo, alguns poros já contêm a etringita massiva e alguma fissuração já foi notada na matriz cimentícia aos 6 meses, sendo responsável pelas expansões atingidas e que já suplantam o limite adotado para esse método. Porém, essas características se mostram menos expressivas quando comparadas àquelas observadas no concreto testado no método brasileiro, indicando um estágio menos avançado do processo de desenvolvimento da DEF. Sendo assim, pela disponibilidade de íons na solução dos poros do concreto, que sabidamente ainda existe, o agravamento desse quadro deverá ocorrer em idades mais avançadas, e até 365 dias.

Alshamaa et al. (2016) verificaram através de MEV a evolução de concretos afetados por DEF, em que apresentou neoformações massivas de cristais, principalmente na interface entre o agregado e a pasta de cimento, contendo também fissuras na matriz cimentícia. Os estudos realizados por Sun et al. (2009) e Zhang et al. (2016) corroboram os resultados apresentados em relação às formações de cristais de etringita na interface pasta/agregado, e com evoluções crescentes ao longo do tempo.

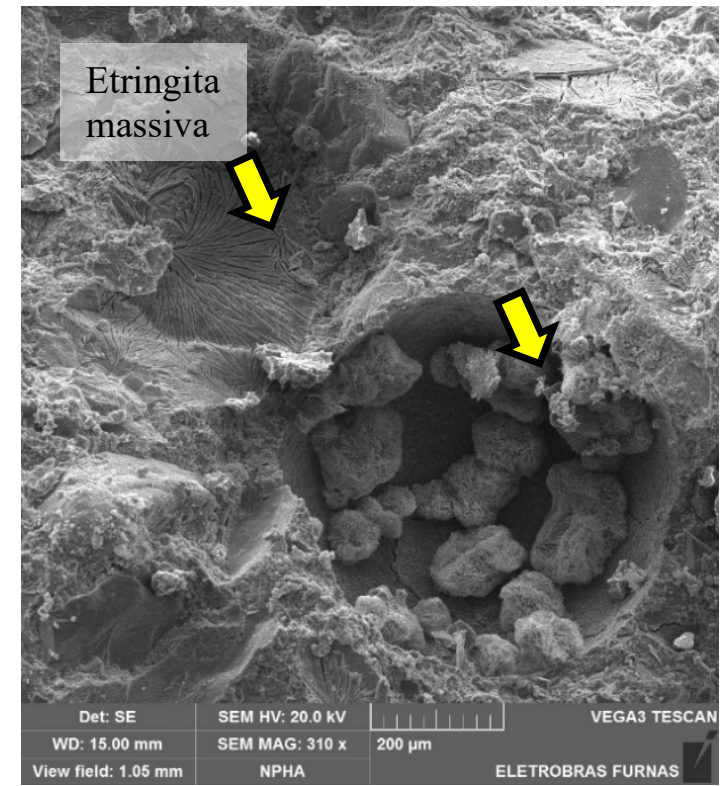

a)

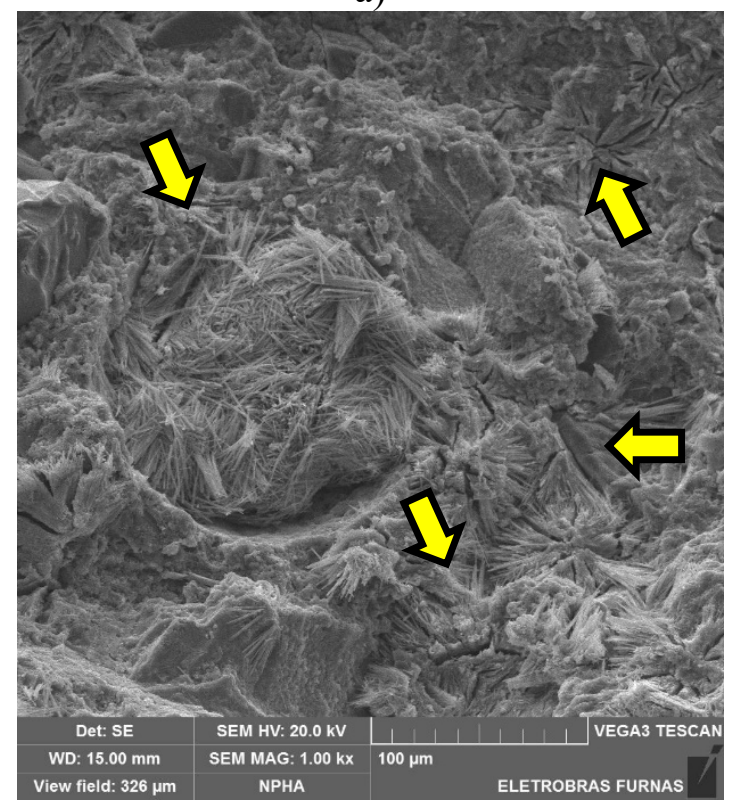

n)

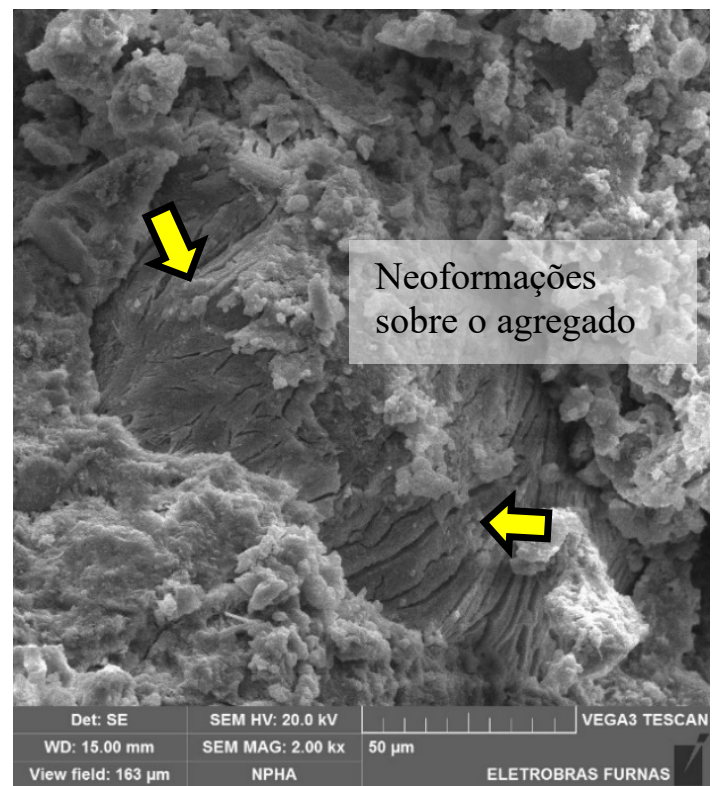

b)

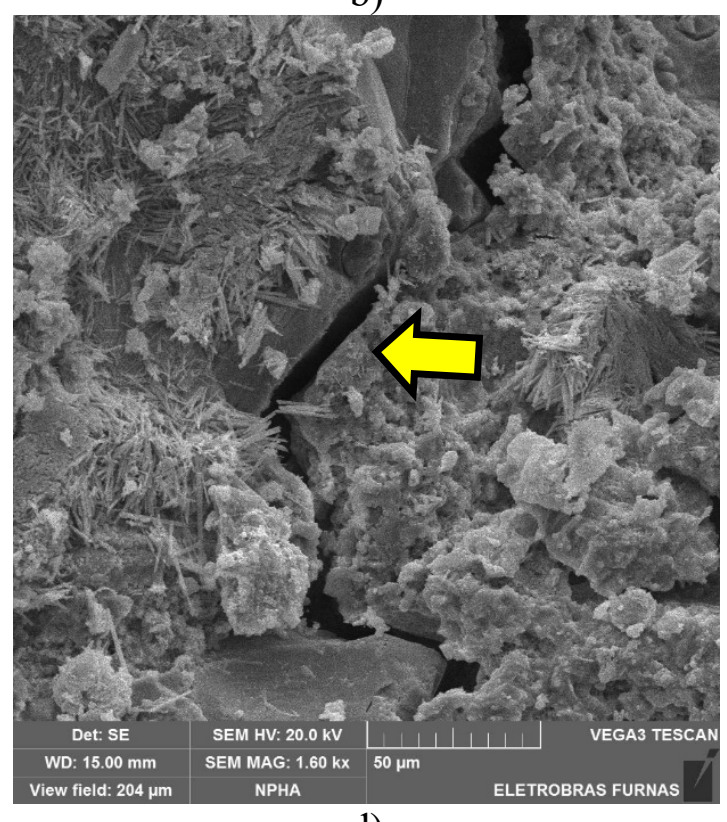

d) 
Figura 8. Concreto submetido ao método de ensaio Brasileiro - Micrografias obtidas por MEV: a)

Poros com neoformação de etringita na forma massiva e aglomerada; b) Agregado totalmente recoberto por cristais de etringita massiva indicando fragilização na ZT; c) Grande concentração de etringita dispersa na pasta e em poro; d) Fissura gerada pela expansão da DEF.

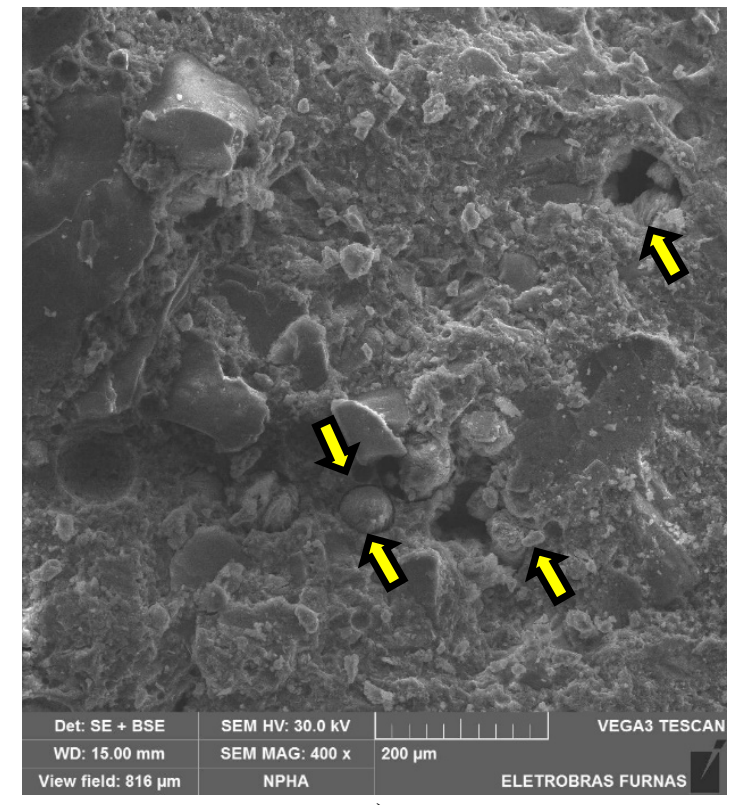

a)

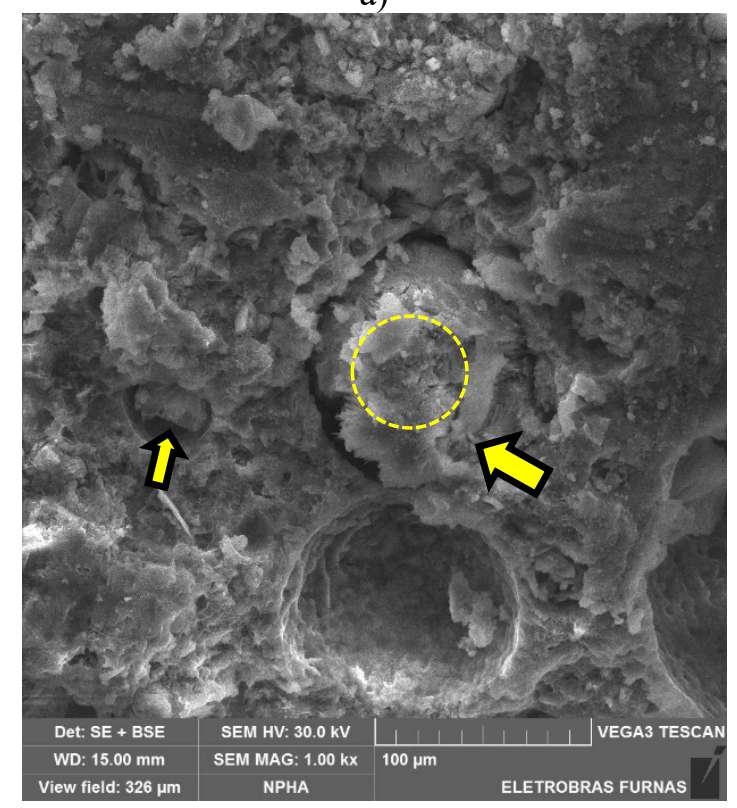

c)

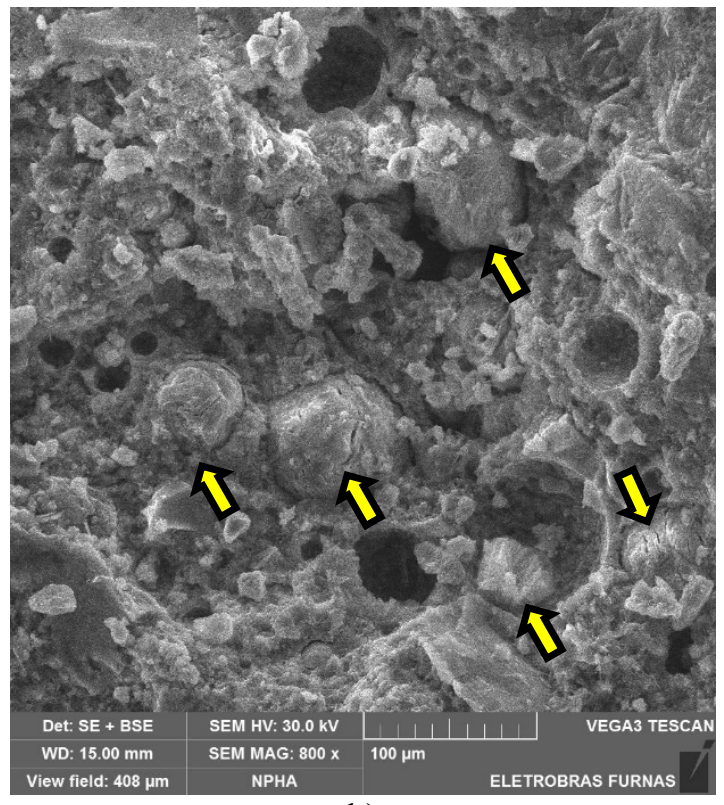

b)

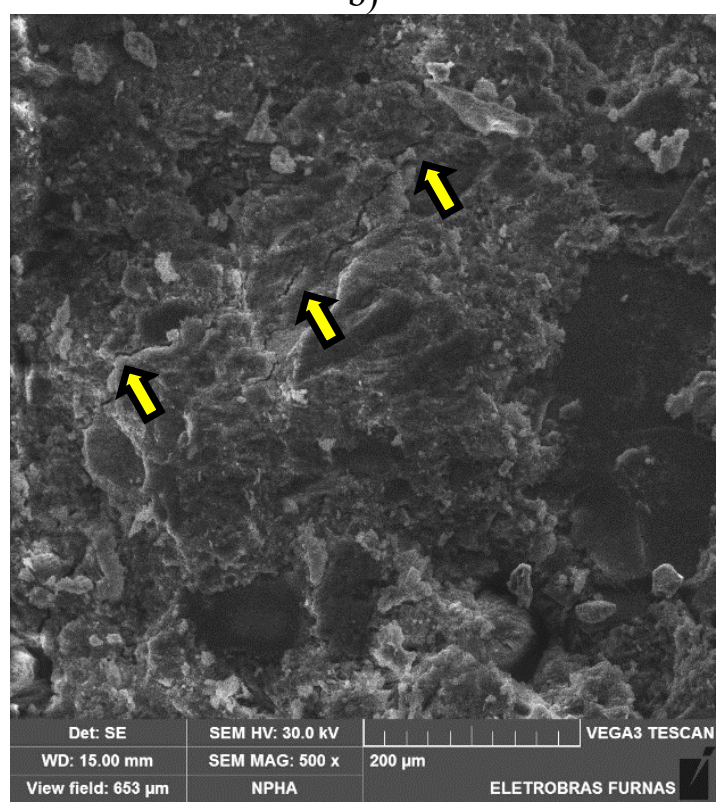

d)

Figura 9. Concreto submetido ao método de ensaio Francês - Micrografias obtidas por MEV: a) Vista geral da amostra indicando alguns poros vazios e outros preenchidos por etringita; $b$ ) Detalhe do tamponamento de alguns poros pelas neoformações; c) Imagem mais ampliada de poro com etringita comprimida (ver espectro onde indicado); d) Microfissura gerada na pasta pela DEF.

Todas as neoformações apresentadas nas micrografias foram avaliadas também por microanálise (EDX), sendo confirmado o composto etringita, tendo com o padrão de espectro apresentado na Figura 10. 


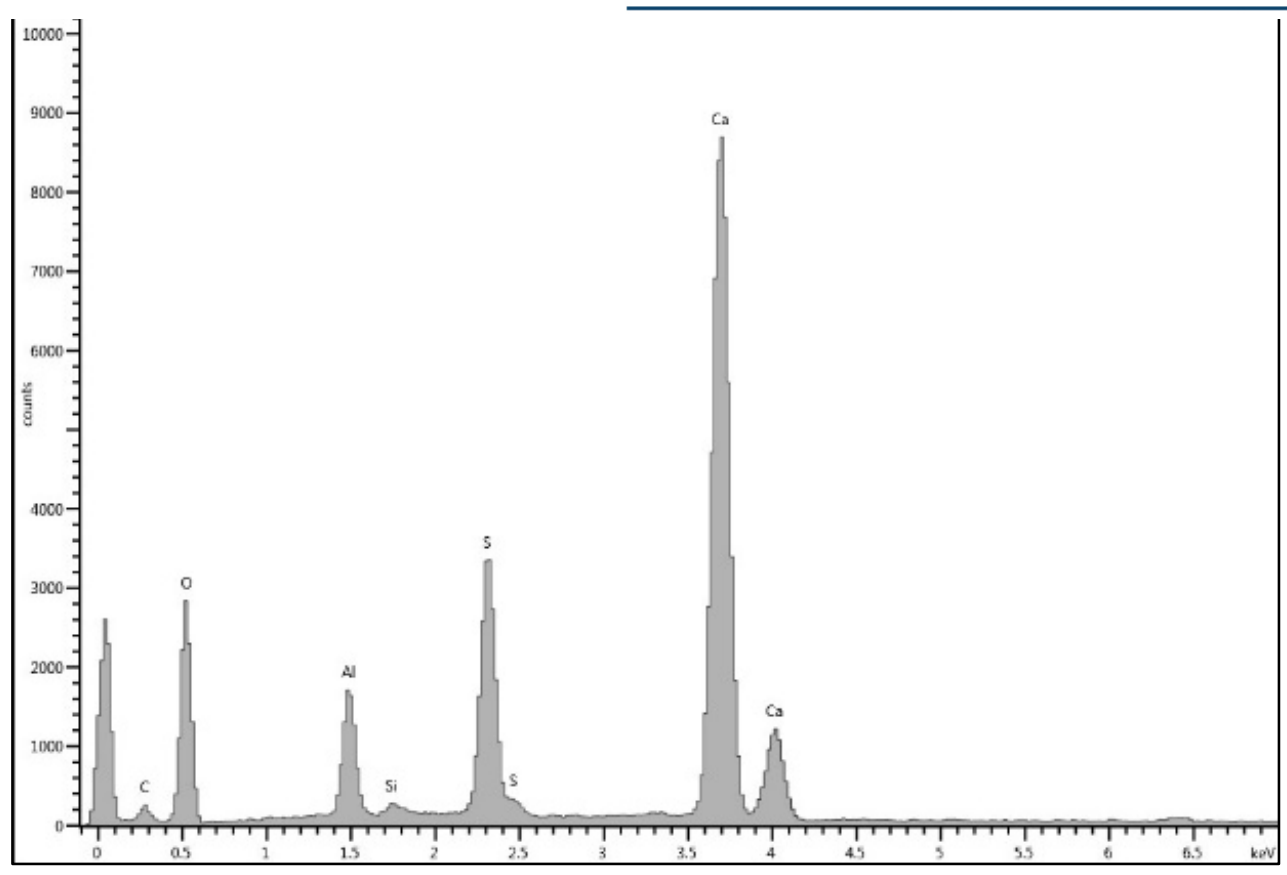

Figura 10. Espectro característico da etringita indicada na Figura 9c.

\section{CONCLUSÕES}

A partir dos resultados obtidos foi possível concluir que a avaliação do potencial de expansão ocasionado pela DEF em prismas de concreto moldados em laboratório, com seis meses de ensaio, foi mais adequada quando do emprego do método brasileiro, na presença de um cimento tipo CP V-ARI, sem adições.

Os concretos submetidos ao método brasileiro de ensaio apresentaram redução significativa em suas propriedades mecânicas devido à fissuração ocasionada pela DEF, corroborada pelas elevadas expansões atingidas, da ordem de $1 \%$ aos seis meses de ensaio, e pela microestrutura dos concretos avaliada. $\mathrm{O}$ método francês indicou valores menores de expansão aos seis meses $(0,05 \%$, em média), porém acima do limite permitido, indicando também potencial para a $\mathrm{DEF}$, apesar de ainda não terem sido observadas reduções nas propriedades mecânicas avaliadas.

As diferenças nos resultados obtidos observadas entre os dois métodos aplicados devem-se ao critério empregado no ambiente de exposição ao longo do tempo. Enquanto no método brasileiro a exposição é em um ambiente fixo, e com temperatura de $38^{\circ} \mathrm{C}$, no método francês são promovidos inicialmente ciclos e, na sequência, exposição a $20^{\circ} \mathrm{C}$, ambos em imersão em água. Além das diferenças nas temperaturas empregadas, o processo cíclico pode influenciar promovendo lixiviação dos componentes químicos da solução dos poros, afetando a cinética das reações. Nesse quesito, devem ser realizadas análises suplementares e o acompanhamento da água de imersão e da solução dos poros dos concretos, por meio de técnicas específicas, de forma a elucidar esses comportamentos.

Ambos os métodos atingiram expansões superiores ao limite aceitável (0,04\%) para garantir a durabilidade das estruturas frente à DEF. Entretanto, através das análises microestruturais foi confirmado que, para o mesmo período de ensaio, os concretos submetidos ao método brasileiro apresentaram avançada formação de DEF nos poros e na matriz cimentícia, ao contrário daqueles submetidos ao método francês, com poucas formações da etringita massiva até a idade de seis meses avaliada. Portanto, esse método deve ter a duração de um ano para delinear melhor o comportamento do concreto, conforme preconizado no próprio procedimento em pauta; por outro lado, o método brasileiro indicou a sua aplicabilidade para um tempo mais reduzido, de seis meses, se mostrando bastante promissor. 
Este trabalho faz parte do projeto de P\&D Metodologias e infraestrutura tecnológica para aperfeiçoamento das avaliações de confiabilidade e otimização de empreendimentos de energia PD.0394-1504/2015, regulamentado pela Agência Nacional de Energia Elétrica - ANEEL, desenvolvido pela Eletrobras FURNAS e INSTITUIÇÃO

\section{REFERÊNCIAS}

Associação Brasileira de Normas Técnicas. (2011). NBR 7222: Concreto e argamassa Determinação da resistência à tração por compressão diametral de corpos de prova cilíndricos. Rio de Janeiro.

Associação Brasileira de Normas Técnicas. (2018). NBR 5739: Concreto - Ensaio de compressão de corpos de prova cilíndricos. Rio de Janeiro.

Bronholo, J. (2020). "Estudo do ataque individual e misto de DEF e RAA e de seus efeitos deletérios nas propriedades físico-químicas e mecânicas de concretos e argamassas de cimento Portland Pozolânico e de alta resistência" Dissertação de Mestrado, Instituto de Engenharia do Paraná, p. 216.

Day, R. L. (1992). The effect of secondary ettringite formation on the durability of concrete: a literature analysis.

Duggan, C. R., Scott, J. F. (1987) Proposed new test for alkali-aggregate reactivity, Canadian National Railways, Technical Research Report, Montreal (Canada).

Giannini, E. R., Sanchez, L. F. M., Tuinukuafe, A., Folliard, K. J. (2018), Characterization of concrete affected by delayed ettringite formation using the stiffness damage test. Construction and Building Materials. 162:253-264.

Gillott, J. E., Grabowski, E., Jones, T. N., Quinn, T., Scott, J. F., Duggan, C.R. (1989). “Mechanism of expansion in rapid test method for alkali-aggregate reaction". Regress Report 1, Dept. of Civil Eng., University of Calgary, Calgary (Canada).

Godart, B. (2017), Pathology, assessment and treatment of structures affected by delayed ettringite formation. Structural Engineering International, 27:362-369.

Godart, B.; Divet L. (2013). "Lessons learned from structures damaged by delayed ettringite formation and the French prevention strategy". Fifth international conference on Forensic Engineering, Paris (France).

Grabowski, E., Czarnecki, B.; Gillott, J. E.; Duggan, C. R.; Scott, J. F. (1992). Rapid test of concrete expansivity due to internal sulfate attack, ACI Mater. 89:469-480.

Hasparyk, N. P., Kuperman, S. C. (2019). "Deterioração do concreto por reações expansivas" in: XXXII - Seminário Nacional de Grandes Barragens - SNGB. - Comitê Brasileiro de Barragens CBDB. Salvador (Brazil).

Hasparyk, N. P., Sanchez, L. (2021), "SDT - Método de Ensaio para a Determinação do Índice de Dano de Rigidez (SDI) e Índice de Deformação Plástica (PDI) em Concretos - Instrução Técnica ITDSBE001". Goiânia: Ed. Furnas.

Hasparyk, N. P., Schovanz, D., Kuperman, S. (2020), "Método de Ensaio para a Avaliação do Potencial de Ocorrência da Etringita Tardia (DEF) em Concreto - Instrução Técnica ITGSTE004". Goiânia: Ed. Furnas.

Jebli, M., Jamin, F., Pelissou, C., Lhopital, E., \& Youssoufi, M. S. E. (2021). Characterization of the expansion due to the delayed ettringite formation at the cement paste-aggregate interface. Construction and Building Materials, 289. https://doi.org/10.1016/j.conbuildmat.2021.122979

Kchakech, B., Martin, R. P., Omikrine-Metassi, O., Renaud, J. C., Baron, L., Toutlemonde, F. (2016). 
with deläyed étringite formation” in: Proceèding of 15 International Conference ó Alkali Aggregate Reaction in Concrete - 15th ICAAR, São Paulo (Brazil).

Lavaud, S., Divet, L., Linger, L., Boutillon, L., Projets, V. C. G., \& Rueil-Malmaison, F. (2013), Delayed ettringite formation-outcomes of laboratory investigations carried out on protective treatments.

LCPC - Laboratoire Central des Ponts et Chaussées. (2007). "Réactivité d'un béton vis-à-vis d'une réaction sulfatique interne", Techniques et méthodes des laboratoires des Ponts et Chaussées, Méthode d'essai des LCPC n 66 , Paris.

Martin, R., Sanchez, L., Fournier, B., Toutlemonde, F. (2017), Evaluation of Different Techniques for the Diagnosis \& Prognosis of Internal Swelling Reaction (ISR) Mechanisms in concrete. Construction and Building Materials. 156:956-964. https://doi.org/10.1016/j.conbuildmat.2017.09.047

N. Leklou (2008) "Contribution to the Knowledge of Internal Sulphate Reaction" PhD Thesis, L’Université Paul Sabatier Toulouse.

Pavoine, A., Divet, L., Fenouillet, S. (2006), A concrete performance test for delayed ettringite formation: Part I optimisation. Cement and Concrete Research, 36:2138-2143.

Sanchez, L. F. M.; Drimalas, T.; Fournier, B.; Mitchell, D.; Bastien, J. (2018), Comprehensive damage assessment in concrete affected by different internal swelling reaction (ISR) mechanisms. Cement and Concrete Research, 107:284-303.

Schovanz, D. (2019). "Estudo da formação da etringita tardia (DEF) em concretos com cimento Portland pozolânico e de alta resistência". Dissertação de Mestrado, Faculdade IMED, p. 180.

Schovanz, D., Tiecher, F., Hasparyk, N. P., Kuperman, S., Lermen, R.T. (2021), Evaluation of delayed ettringite formation through physical, mechanical, and microstructural assays. ACI Materials Journal. 118:101-109. https://doi.org/10.14359/51728282

Schovanz, D., Tiecher, F., Hasparyk, N. P., Kuperman, S. C. (2019). "Influência da formação da etringita tardia (DEF) na microestrutura e propriedades mecânicas do concreto" in: IBRACON (Ed.), $61^{\circ}$ Congresso Brasileiro do Concreto, Fortaleza (Brazil).

Shamaa, M., Lavaud, S., Divet, L., Colliat, JB., Nahas, G., Torrenti, J. M. (2016), Influence of limestone filler and of the size of the aggregates on DEF. Cement and Concrete Composites. 71:175180. https://doi.org/10.1016/j.cemconcomp.2016.05.007

Sun, H., Jain, R., Nguyen. K., Zuckerman, J. (2009), Sialite technology - Sustainable alternative to portland cement. Clean Technologies and Environmental Policy. 12:503-516.

Torres, I. F.; Andrade, T. (2016), Análise de risco da formação de etringita tardia em blocos de fundação na Região Metropolitana de Recife/PE-Brasil. RIEM - IBRACON Structures and Materials Journal, v. 9, n. 3 .

Zhang, N., Li, H., Peng, D., Liu, X. (2016), Properties evaluation of silica-alumina based concrete: Durability and environmental friendly performance. Construction and Building Materials. 115:105113. https://doi.org/10.1016/j.conbuildmat.2016.04.043 